\title{
Application of Teager-Kaiser energy operator to the analysis of the water shaft bearings
}

\author{
Jędrzej Blaut ${ }^{1}$, Tomasz Korbiel ${ }^{2}$, Wojciech Batko ${ }^{3}$ \\ University of Science and Technology, Kraków, Poland \\ ${ }^{1}$ Corresponding author \\ E-mail: ${ }^{1}$ blaut@agh.edu.pl, ${ }^{2}$ tkorbiel@agh.edu.pl, ${ }^{3}$ batko@agh.edu.pl \\ Received 7 September 2017; accepted 15 September 2017 \\ DOI https://doi.org/10.21595/vp.2017.19080
}

Check for updates

\begin{abstract}
A novel vibration-based monitoring technique for shaft bearings in seagoing vessels is presented in this paper. Described observation of the lubricated wedge stability was carried out using test fixture. The analysis of results is based on an original method which put Energy Operator of Teager Kaiser to use. Analysis results were compared with the method built on trajectory of the centre shaft axle observation.
\end{abstract}

Keywords: shaft pipe, water lubricated rubber bearings, Teager-Kaiser energy operator, TKEO, fault diagnosis.

\section{Introduction}

Stern tubes are used to house the bearings and seal the shaft. The design of a stern tube depends on the lubrication agent. At the beginning, stern tubes were equipped with water lubricated lignum vitae (a dense wood) and the system of bearings was open. Lignum vitae is a very hard, heavy (density: $1230 \mathrm{~kg} / \mathrm{m}^{3}$ ), abrasion-resisting wood. The stern tubes with guaiacum bearings were sealed only between the tube and the engine.

The World War I caused the lack of guaiacum and changes of the structure of bearings. Structures of lubricated with oil bearings became popular requiring the additional sealing. Frequent leaks of oils from lubrication systems to water bodies caused changes in the law.

At present, the restrictive law is a factor encouraging ship-owners to applying eco-friendly bearings lubricated with water. Modern bearings lubricated with water time of exploitation is comparable to bearings lubricated with oil. The longevity of stern tube in off shore navigation (not open sea) is very limited when the water is impurity by high content of sand or mud.

A use of composite bearings lubricated with environmental friendly liquid is possible in the close system without the risk of contamination of the natural [1].

\section{Experiments on laboratory test fixture}

Tests were conducted in test fixture, which was prepared for using the composite bearing lubricated with water used in the sea shipping. Multi-groove aft bearing with the diameter of $20 \mathrm{~mm}$ made of rubber, planted on the bronze hub was built. The bearing was sealed on both sides and filled with water. The flow of water is forced with the help of water pump. Character 1 is describing the created test fixture.

During the experiment, the stand was loaded with the force of $160 \mathrm{~N}$ in the horizontal plane by the bearing loading system (6, Fig. 1). This corresponds to the shaft deflection by $1,2 \mathrm{~mm}$. The experiment involved examining the trajectory of shaft rotation axis. The experiment was conducted at various speeds, from 300 to 6800 RPM, in 500 RPM increments. The recorded displacement signal was analyzed.

\section{Analyses}

According to the literature [2, 3], there are three hydrodynamic states in plain bearings: normal operation, small oil vortexes, large oil vortexes (Fig. 2). 


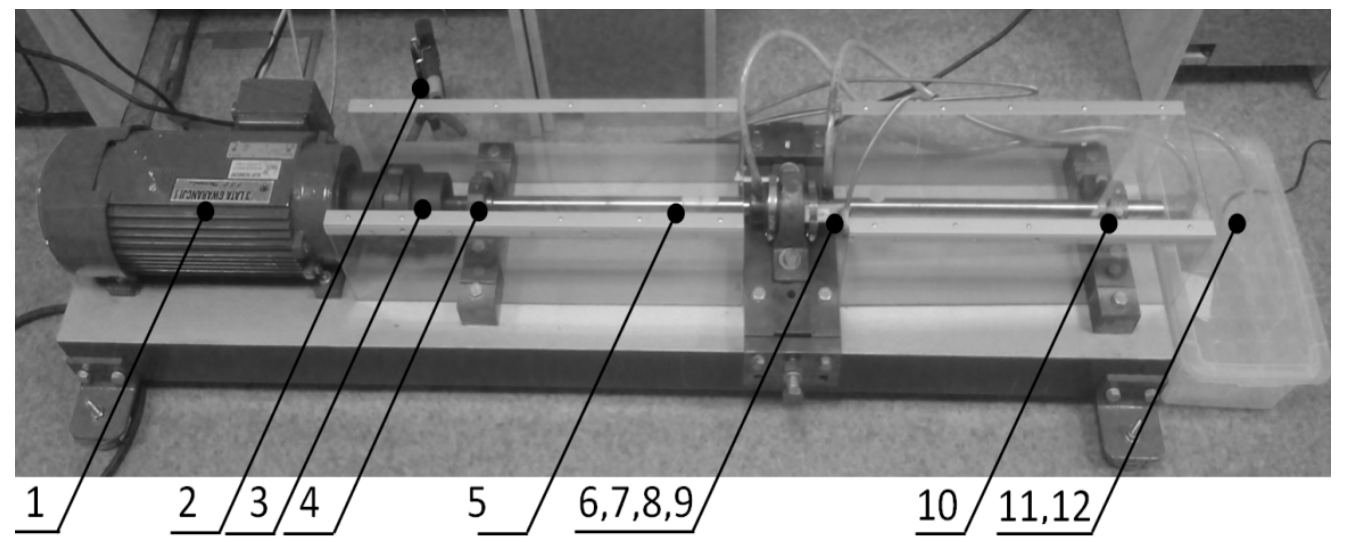

Fig. 1. Test stand, 1 - motor with speed control, 2 - tachometer, 3 - coupling, 4, 10 - rolling bearing, 5 - shaft, 6 - plain bearing, 7 - bearing loading system, 8 - lubricant delivery and removal valves, 9 - eddy-current sensors, 12 - lubricant tank

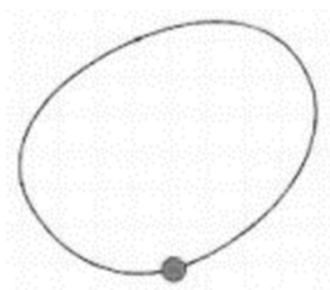

a) Stable region 1 Phase marker

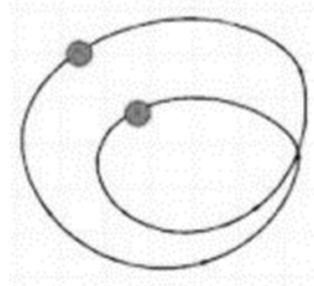

b) Oil whirl region 2 Phase marker

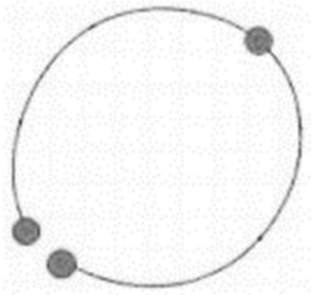

c) Oil whip region 3 Phase marker

Fig. 2. Classification of hydrodynamic instabilities in the system and introduction of diagnostic determinants [2]

Trajectories can be determined by correlation of two signals from sensors (9, Fig. 1). A new method is based on the observation of value of Teager-Kaiser Energy Operator (TKEO) during work of shaft [4-6]. It can be done offline and online also.

TKEO is defined in the continuous form as [7]:

$\Psi(x(t))=\dot{x}^{2}(t)-x(t) \ddot{x}(t)$.

A work of the aft bearing during the changing rotation speeds was analyzed. An amplitude of the TKEO value was being examined. The growth of the TKEO fluctuation rotational trajectories matching them were analyzed. A relation of the Teager-Kaiser Energy Operator (TKEO) fluctuation growth was demonstrated in case of loss of the stability of the work of the bearing.

\section{Results}

The whole signal of moving was examined (separately $x$ and $y$ ) looking up the sudden changes of amplitude of TKEO. The value of amplitude was determined for every turnover and then created was a diagram of TKEO amplitude to turnovers. An exemplary diagram (rotational speed of $2800 \mathrm{rpm}$ for the signal of moving in y axis) presented in Fig. 3.

The TKEO value depends on the trajectory, the speed and on the acceleration, according to the model (1), indirectly depends also on the shape of the trajectory. The changes of the values of amplitude about $50 \%$ were observed. Fig. 4 shows the trajectory of turn 104 and 105 (for which amplitude TKEO carries out 2200) for the comparison in Fig. 5 presented the later turns of 107 and 108 for which the amplitude carries out 3200. Presented do not differ substantially from each 
other, however the value of amplitude TKEO differs about $50 \%$.

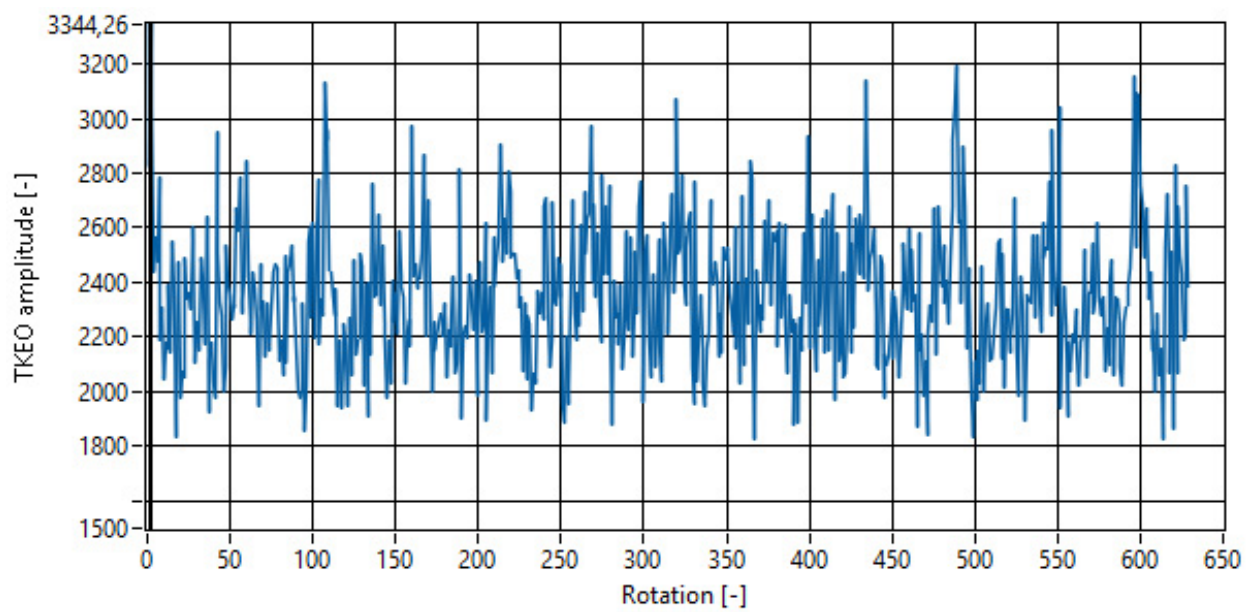

Fig. 3. Value of the TKEO amplitude for next turnovers of the rotor

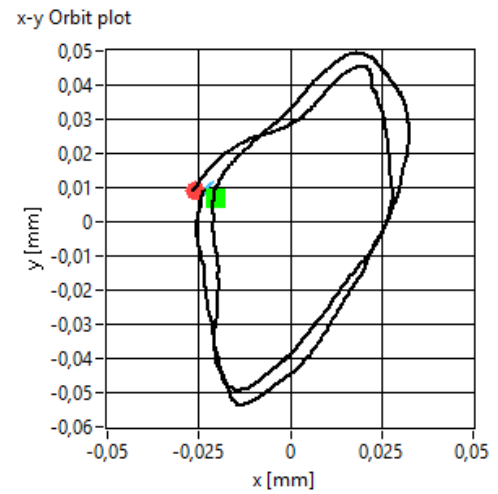

Fig. 4. Trajectory of turnover 104 and 105

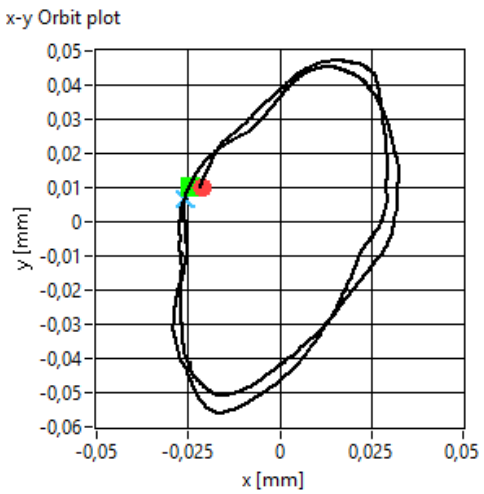

Fig. 5. Trajectory of turnover 104 and 105

Table 1. Ccomparison of rotation speed and TKEO value

\begin{tabular}{|c|c|c|c|c|}
\hline RPM & $\begin{array}{c}\text { Detected } \\
\text { frequency }\end{array}$ & $\begin{array}{c}\text { Classification of } \\
\text { hydrodynamic instabilities }\end{array}$ & $\begin{array}{c}\text { TKEO mean } \\
\text { amplitude signal } x\end{array}$ & $\begin{array}{c}\text { TKEO mean } \\
\text { amplitude signal } y\end{array}$ \\
\hline 300 & 5,01 & Stable region & 221 & 188 \\
\hline 800 & 13,39 & Stable region & 277 & 230 \\
\hline 1300 & $21,5\left(43^{*}\right)$ & Stable region & 2540 & 1821 \\
\hline 1800 & 30 & Stable region & 2492 & 2098 \\
\hline 2300 & - & - & - & 2412 \\
\hline 2800 & 46,69 & Stable region & 3326 & 4172 \\
\hline 3300 & 55,01 & Stable region & 6185 & 4275 \\
\hline 3800 & 63,34 & Stable region & 7249 & 5537 \\
\hline 4300 & 61,7 & Stable region & 9247 & 34174 \\
\hline 4800 & $80(160 *)$ & Stable region & 40211 & 9875 \\
\hline 5300 & 88,35 & Stable region & 14283 & 12197 \\
\hline 5800 & 96,65 & Stable region & 18325 & 55830 \\
\hline 6300 & $105(210,02 *)$ & Stable region & 83884 & 18301 \\
\hline 6800 & 113 & Stable region & 27931 & \\
\hline
\end{tabular}

During experiment, hydrodynamic instabilities weren't noticed. An observed increase in TKEO amplitude along with the rotation speed is shown in Table 1. During measurements: 1300, 
4800,6300 an abnormal work of the tachometer was noticed.

\section{Conclusions}

The conducted experiment proved dependence of value of amplitude of TKEO on circulating speed. Significant influence of the shape of trajectory on the value of the amplitude TKEO was not observed. Abnormal work of tachometer resulted in the changes of parameters of filtration of signal. It paid attention to the relation of the width of selected band of the filtration to the value of the TKEO amplitude. Examinations letting the hydrodynamic stability observation in order to examine its influence on the value of the TKEO amplitude were planned.

\section{References}

[1] Myynarczak A. A case of bearings seizing in shaft pipe. Scientific Journal of Polish Naval Academy, 2014, https://doi.org/10.5604/0860889X.1139633.

[2] Kiciński J. Model based diagnostics - today and tomorrow. Diagnostyka, Vol. 30, 2004, p. 241-248.

[3] Blaut J., Korbiel T., Batko W. Application of the teager-kaiser energy operator to detect instability of a plain bearing. Diagnostyka, Vol. 17, 2016, p. 99-105.

[4] Blaut J., Batko W., Korbiel T. Using Teager Kaiser energy operator for detection state of hydrodynamic fluid film in bearing monitoring. 43th Nationwide Symposium on Diagnostics of Machines, Wisła, 2016.

[5] Henríquez P. Application of Teager-Kaiser energy operator to the analysis of degradation of a helicopter input pinion bearing. The International Conference Surveillance, 2011.

[6] Li H., Fu L., Zhang Y. Bearing faults diagnosis based on teager energy operator demodulation technique. Measuring Technology and Mechatronics Automation, 2009.

[7] Kaiser J. On a simple algorithm to calculate the 'energy' of a signal. International Conference on Acoustics, Speech, and Signal Processing, 1990. 